\title{
Early Onset Multiple Sclerosis: More Emphasis on Early Treatment
}

Multiple sclerosis (MS) is an immune-mediated neurodegenerative disorder of the human central nervous system (CNS) that typically affects young adults. This enigmatic and destructive disorder of both white matter and gray matter of CNS affects individuals with predisposing genetic background and probably requires a provoking environmental factor, such as exposure to a viral agent, to initiate the disease process [1-3]. In the United States, MS is the leading cause of neurological disability in young individuals. Clinically, MS most often follows a relapsing-remitting course during which patients experience various clinical symptoms, usually followed by periods of remission. The neuropathogenesis of MS consists of two parallel destructive arms: inflammatory demyelination and neurodegeneration. During the last two decades, we have learned much about both aspects of the pathogenesis of MS. This expansion of our knowledge had led to development of immunomodulatory agents for more effective management of this previously untreatable disease.

Despite these enormous advances in our understanding of the pathogenesis of MS in adult patients, much less is known about how MS affects the very young. Traditionally, MS has been regarded as a "rare" disease of childhood. A review of MS literature, however, demonstrates that at least $5 \%$ of MS patients first manifest symptoms prior to age 18 [4,5]. Interestingly, more than $97 \%$ of these patients initially experience a relapsing-remitting disease [6]. Compared to adult patients, diagnosis of pediatric MS poses a challenge due to a higher occurrence of other agerelated white matter diseases in this age group. In addition, children with MS develop disability at an earlier age than adults.

Currently, immunomodulating agents are recommended for management of adult patients with MS. However, until recently, there has been no standard treatment for very early onset of MS.
Banwell et al. [7] retrospectively reviewed safety and tolerability of interferon-beta-1b in a cohort of 43 children and adolescents. The group treated with IFN-beta-1b for a mean of 29.2 months tolerated treatment well. No safety issues were reported.

In this issue of Journal of Neuropediatrics, Pakdaman et al. [8] report the results of a prospective clinical trial utilizing a suboptimal dose of IFN-beta-1a in children with early onset MS. The investigators randomized 16 consecutive children with early onset relapsing-remitting MS to treatment with IFN-beta-1a 15 mcg IM once weekly or no treatment. Subjects were followed for four years and underwent periodic neurological examination and assessment of safety measures. Study results demonstrated that subjects who were randomized to treatment tolerated suboptimal doses of IFN well with only mild side effects and no significant adverse events. At the end of the trial, the treated subjects had less progression of disability compared to the untreated subjects.

Both of these clinical trials utilizing beta-interferons in patients with early onset MS provide strong evidence for the concept of early treatment approach in this patient group, with the goal of slowing disease progression and delaying onset of disability. The long-term effects of beta-interferons on children who are still maturing demand further assessment, however.

\section{References}

${ }^{1}$ Frohman EM, Racke MK, Raine CS. Multiple sclerosis - the plaque and its pathogenesis. N Engl J Med 2006; 354: 942 - 955

${ }^{2}$ Noseworthy JH, Lucchinetti C, Rodriguez M, Weinshenker BG. Multiple sclerosis. N Engl J Med 2000; 343: 938 -952 
3 Sospedra M, Martin R. Immunology of multiple sclerosis. Annu Rev Immunol 2005; 23: $683-747$

${ }^{4}$ Duquette P, Murray TJ, Pleines J, Ebers GC, Sadovnick D, Weldon P, Warren S, Paty DW, Upton A, Hader W et al. Multiple sclerosis in childhood: clinical profile in 125 patients. J Pediatr 1987; 111: 359-363

${ }^{5}$ Ghezzi A, Deplano V, Faroni J, Grasso MG, Liguori M, Marrosu G, Pozzilli C, Simone IL, Zaffaroni M. Multiple sclerosis in childhood: clinical features of 149 cases. Mult Scler 1997; 3: $43-46$
${ }^{6}$ Boiko A, Vorobeychik G, Paty D, Devonshire V, Sadovnick D. Early onset multiple sclerosis: a longitudinal study. Neurology 2002; 59: $1006-1010$

7 Banwell B, Reder AT, Krupp L, Tenembaum S, Eraksoy M, Alexey B, Pohl D, Freedman M, Schelensky L, Antonijevic I. Safety and tolerability of interferon beta-1b in pediatric multiple sclerosis. Neurology 2006; 66: $472-476$

8 Pakdaman H, Fallah A, Sahraian MA, Pakdaman R, Maysami P. Treatment of early onset multiple sclerosis with suboptimal dose of interferon beta 1a. Neuropediatrics 2006; 37: 257-260 